\title{
A Network Pharmacology-Based Approach to Investigating the Mechanisms of Fushen Granule Effects on Intestinal Barrier Injury in Chronic Renal Failure
}

\author{
Miaoru Han, Hangxing Yu, Kang Yang, Panying Liu, Haifeng Yan, Zhihua Yang, \\ and Hongtao Yang $\mathbb{B}$ \\ Department of Nephrology, First Teaching Hospital of Tianjin University of Traditional Chinese Medicine, Tianjin, China \\ Correspondence should be addressed to Hongtao Yang; tjtcmht@126.com
}

Received 1 August 2020; Revised 15 February 2021; Accepted 25 February 2021; Published 5 March 2021

Academic Editor: Vahidreza Ostadmohammadi

Copyright (c) 2021 Miaoru Han et al. This is an open access article distributed under the Creative Commons Attribution License, which permits unrestricted use, distribution, and reproduction in any medium, provided the original work is properly cited.

Purpose. Fushen Granule (FSG) is a Chinese medicine prepared by doctors for treating patients with chronic renal failure, which is usually accompanied by gastrointestinal dysfunction. Here, we explore the protective effect of FSG on intestinal barrier injury in chronic renal failure through bioinformatic analysis and experimental verification. Methods. In this study, information on the components and targets of FSG related to CRF is collected to construct and visualize protein-protein interaction networks and drug-compound-target networks using network pharmacological methods. DAVID is used to conduct gene ontology (GO) enrichment analysis and Kyoto Encyclopedia of Genes and Genomes (KEGG) pathway enrichment analysis. Then, it is validated by in vitro experiments. In this study, the human intestinal epithelial (T84) cells are used and divided into four groups: control group, model group, FSG low-dose group, and FSG high-dose group. After the experiment, the activity of T84 cells is detected by a MTT assay, and the expressions of tight junction protein ZO-1, claudin-1, nuclear factor erythroid 2-related factor (Nrf2), heme oxygenase-1 (HO-1), malondialdehyde (MDA), and cyclooxygenase-2 (COX-2) are examined by immunofluorescence and/or western blotting. Results. Eighty-six potential chronic renal failure-related targets are identified by FSG; among them, nine core genes are screened. Furthermore, GO enrichment analysis shows that the cancer-related signaling pathway, the PI3K-Akt signaling pathway, the HIF1 signaling pathway, and the TNF signaling pathway may play key roles in the treatment of CRF by FSG. The MTT method showed that FSG is not cytotoxic to uremic toxin-induced injured T84 cells. The results of immunofluorescence and WB indicate that compared with the control group, protein expressions level of ZO-1, claudin-1, and Nrf2 in T84 cells is decreased and protein expressions level of HO-1, MDA, and COX-2 is increased after urinary toxin treatment. Instead, compared with the model group, protein expressions level of ZO-1, claudin-1, and Nrf2 in T84 cells is increased and protein expressions level of HO-1, MDA, and COX-2 is decreased after FSG treatment. Conclusion. FSG had a protective effect on urinary toxin-induced intestinal epithelial barrier injury in chronic renal failure, and its mechanism may be related to the upregulation of Nrf2/HO-1 signal transduction and the inhibition of tissue oxidative stress and inflammatory responses. Screening CRF targets and identifying the corresponding FSG components by network pharmacological methods is a practical strategy to explain the mechanism of FSG in improving gastrointestinal dysfunction in CRF.

\section{Introduction}

Chronic kidney disease (CKD) is a kind of renal disease featuring chronic renal dysfunction and structural damage. The glomerular filtration rate (GFR) is the best index reflecting overall kidney function [1]. CKD has become a serious global public health problem. There were 1.2 million deaths worldwide in 2017 due to CKD. The mortality of CKD has been rising, and it is predicted that, by 2040 , the number will rise worldwide to 2.2 million conservatively, even up to 4 million $[2,3]$. The renal function of CKD patients gradually decreases due to nitrogen waste accumulation, which is discharged by normal persons over time and eventually progresses to chronic renal failure (CRF), which is always 
accompanied by a progressive decline in glomerular filtration rate and the continuous accumulation of metabolic waste. In recent years, a large number of studies have reported that the accumulation of urinary toxins and the influx of other residual toxins destroy the intestinal epithelial barrier, allowing the entry of intestinal bacteria into the blood, which further aggravates the progression of CRF $[4,5]$, and finally, end-stage renal disease (ESRD) develops. The gut barrier is composed of occludin, ZO-1, and E-cadherin. The expression of claudin-1, occludin, and ZO-1 in the intestinal tissue of five out of six nephrectomized rats was significantly reduced, and there was significant systemic oxidative stress in the rats [6]. In addition, the content of enterotoxins such as para-cresol sulfate (PCS) and indole sulfate (IS) was significantly increased in the serum of CKD patients and long-term dialysis patients, indicating increased intestinal permeability $[7,8]$. In CKD and long-term dialysis patients with damaged intestinal epithelial barriers, intestinal bacterial DNA and uremia-related toxins infiltrate the blood from the intestinal tract, and white blood cells in the blood are activated. This results in the generation of local intestinal cytokines, and further contractions or tight junction protein reactions between endocytic cells occur [9]. This process is a vicious circle, which eventually leads to chronic systemic inflammation. Studies have shown that bacterial DNA fragments from the gut can be detected in the serum of CKD patients whether they are nondialysis patients or dialysis patients $[8,10]$. Therefore, the level of endotoxin from the gut flora in patients with CFR is significantly related to intestinal dysfunction. Lin [11] found that the total serum Is and PC levels in patients with advanced CKD were related to aortic calcification, increased vascular stiffness, and the risk of cardiovascular death. Kim [12] found that the elimination of uremic toxins was an effective treatment for low-turnover osteopathy in CKD patients. Therefore, an important way to improve the nutritional status and quality of life of uremic patients is to regulate intestinal dysfunction. The main symptoms of CRF intestinal injury are abdominal distention, abdominal pain, diarrhea, and constipation. In clinical treatment, antibiotics and gastrointestinal motility drugs are often used. However, the improvement of intestinal mucosal injury by these drugs has not been confirmed, and there is no specific drug to prevent or inhibit intestinal barrier injury.

In China, traditional Chinese medicine is often used to regulate gastrointestinal function and has shown significant effects [13]. Compared to single structure drugs, traditional Chinese medicine has the advantages of multidirectional and multitarget regulation in disease treatment [14]. Through long-term clinical practice, our team found that the traditional Chinese medicine Fushen Granule (FSG) significantly improved the condition of CRF patients complicated with intestinal symptoms [15]. The components of FSG include Salviae miltiorrhizae Radix et Rhizoma (Danshen in Chinese, $30 \mathrm{~g}$ ), Citri Reticulatae Pericarpium (Chenpi in Chinese, $10 \mathrm{~g}$ ), Astragali Radix (Huangqi in Chinese, $15 \mathrm{~g}$ ), Pinelliae Rhizoma (Banxia in Chinese, $15 \mathrm{~g}$ ), root of Chinese clematis (Weilingxian in Chinese, $15 \mathrm{~g}$ ), Radix et Rhizoma Rhei (Shoujun in Chinese, $10 \mathrm{~g}$ ), Euonymus alatus
(Guijianyu in Chinese, $30 \mathrm{~g}$ ), and Angelica sinensis (Danggui in Chinese, $10 \mathrm{~g}$ ). Previous studies have shown that FSG could significantly improve the permeability of the intestinal barrier in peritoneal dialysis rats with CRF. In addition, it also delayed the removal of urinary toxins and water from peritoneal dialysis patients, protected the residual renal function, and improved the nutritional status and clinical syndrome characteristics of peritoneal dialysis patients [16]. Since FSG contains hundreds of compounds and acts on many cell targets, it is difficult to systematically study the mechanism of FSG using conventional methods. Therefore, this study used a network pharmacology strategy to analyze the main active components of FSG from the perspective of a traditional Chinese medicine-compound gene system and explored the mechanism of FSG with multiple components and multiple targets in treating CRF. Then, we subjected the analysis results to experimental verification. In this study, human colon epithelial cells (T84) were cultured in vitro to establish an intestinal barrier injury model induced by urotoxin in CRF and further observe the mechanism of FSG intervention on gut dysfunction induced by urotoxin in CRF.

\section{Materials and Methods}

2.1. Prediction of Traditional Chinese Medicine Targets and Corresponding Active Ingredients. The chemical constituents of Huangqi, Danggui, Xianlingpi, Chenpi, Banxia, Shujun, Danshen, and Guijianyu were identified using the Chinese Medicine System Pharmacology Database and Analysis Platform (TCMSP) and related literature (http://tcmspw. com/tcmsp.php) [17]. On this basis, this study combined the oral bioavailability ( $\mathrm{OB} \geq 30 \%$ ) of chemical ingredients and drug-likeness ( $D L \geq 0.18$ ) as the criteria for screening the active compounds in FSG and identifying the target protein of the corresponding compound. The obtained target protein name was input into the Uniprot (https://www.uniprot. org/) database [18], the species was limited to humans, and all the input target names were corrected to the gene name of the target for the subsequent related analysis.

2.2. Prediction of Chronic Renal Failure-Related Targets. The National Center for Biotechnology Information (NCBI) (https://www.ncbi.nlm.nih.gov/) [19] database is used to provide information on drugs, targets, and drugtargeted diseases and pathways. The Diskenet (https:// www.diskenet.org/) [20] database is a comprehensive gene disease association (GDA) relational database. It provides the latest information on human diseases. The Drugbank (https://www.drugbank.ca/) [21] database provides comprehensive information about western medicine molecules and their mechanisms, including their chemistry, pharmacology, interaction information, and targets. The Therapeutic Target Database (TTD) (http://db.idrblab.net/ttd/) [22] provides information on drugs, targets, diseases, and pathways targeted by drugs. In these four databases, "chronic renal failure" was entered to obtain CRF-related target genes. 
2.3. Intersection of Drug Targets and Disease Targets. A Venn diagram (http://bioinformatics.psb.ugent.be/webtools/Venn/) was drawn, and Wayne diagram software was used to analyze the two datasets online to find the intersecting genes in the two datasets.

\subsection{Construction of Drug-Target-Disease Interaction Network.} The Protein-Protein Interaction Networks Functional Enrichment Analysis (STRING) database provides information on predicted and experimental protein-protein interactions [23]. STRING online (https://string-db.org/cgi/input.pl) was used to construct a protein-protein interaction (PPI) network from the intersection of drug targets and disease targets. The database uses the confidence range of the data scores to define PPI (low confidence is defined by scores of $>0.15$; medium confidence by scores of $>0.4$; and high confidence by scores of $>0.7$ ). Then, the Merge function of Cytoscape software (Cytoscape_v3.2.1) [24] was used to construct a traditional Chinese medicine-compound-target network: (1) the intersecting gene and the corresponding Chinese medicine compound were used to establish a PPI network of the Chinese medicine compound; (2) a traditional Chinese medicine-target PPI network was constructed by connecting the known intersecting genes with their corresponding traditional Chinese medicine; (3) the two networks (1) and (2) were intersected to construct a drugcompound-target pathway network.

2.5. Gene Ontology Analysis. Gene ontology (GO) analysis was carried out for the intersecting genes of the drug targets and disease targets using the Database for Annotation, Visualization, and Integrated Discovery (DAVID) database (https://david.ncifcrf.gov/). The DAVID database integrates various types of database resources. The improved Fisher accurate test algorithm was used to analyze the function and path enrichment of differential genes in the gene set, and the $P$ value and false discovery rate (FDR) were provided for the rich set analysis results [25]. GO enrichment analysis clarifies the role of traditional Chinese medicine compoundtarget proteins in gene function, including the function of three modules: biological processes (BP), molecular function (MF), and cell composition (CC) [26].

2.6. Kyoto Encyclopedia of Genes and Genomes Pathway Enrichment Analysis. Similarly, through the DAVID database (https://david.ncifcrf.gov/) for the enrichment analysis of drug-disease intersection protein targets, KEGG pathway enrichment analysis not only provides an annotation of pathway functions for a given gene set but also provides pathway enrichment analysis [27]. For the multiple test correction of the significant $P$ value in enrichment analysis, the enrichment analysis adopts the algorithm designed by the hypergeometric algorithm and uses the Benjamini-Hochberg correction method. Finally, through the role of the target in the pathway connections, the relevant pathway is obtained [28].

\section{Experimental Verification}

\subsection{Experimental Materials and Methods}

3.1.1. Experimental Cell Lines. The human colon epithelial T84 cell line was purchased from the American Standard Biological Collection Center ATCC (LOT: 62783415).

3.1.2. Experimental Animals. Specific pathogen-free (SPF) male Sprague-Dawley (SD rats), weighing 180-220 g, were purchased from Beijing Viton Lihua Experimental Animal Technology Co., Ltd.

3.1.3. Drug Preparation. FSG was composed of Huangqi 15 g, Danggui 10 g, Xianlingpi 15 g, Chenpi 10 g, Banxia 15 g, Danshen $30 \mathrm{~g}$, Shuju $10 \mathrm{~g}$, and Guijianyu $30 \mathrm{~g}$. The abovementioned traditional Chinese medicine decoction-free granules were provided by the First Affiliated Hospital of Tianjin University of Traditional Chinese Medicine.

3.1.4. Preparation of FSG Drug-Containing Serum. Eighty $\mathrm{SD}$ rats were adaptively fed for one week and randomly divided into three groups: the blank serum group, the lowdose FSG intragastric group, and the high-dose FSG intragastric group. According to the clinical equivalent dose between humans and rats, the rat dose was calculated as $9 \mathrm{~mL} \mathrm{~kg}^{-1} \mathrm{~d}^{-1}$. The low dose was given as $4.5 \mathrm{~mL} \mathrm{~kg}^{-1} \mathrm{~d}^{-1}$, and the high dose was $18 \mathrm{mLkg}^{-1} \mathrm{~d}^{-1}$. The blank serum group was gavaged with normal saline once a day for seven consecutive days with free access to diet. Anesthesia was performed 2 hours after the last gavage. Blood, collected from abdominal aorta of anesthetized rats after 2 hours, was centrifuged to get supernatant. The complement was inactivated by water bath at $56^{\circ} \mathrm{C}$ for $30 \mathrm{~min}$, followed by removal of bacteria through microporous filtration membrane, and then the serum was packed and frozen at $-80^{\circ} \mathrm{C}$.

3.1.5. Cell Culture Treatments. Human colon epithelial T84 cells were cultured in $50 \mathrm{U} \mathrm{L}^{-1}$ penicillin streptomycin ${ }^{-1}$ Dulbecco's modified Eagles Medium (DMEM)/F12 medium containing 10\% fetal bovine serum (FBS; Hyclone, USA) and $50 \mathrm{U} \mathrm{L}^{-1}$ penicillin and streptomycin in $5 \% \mathrm{CO}_{2}$ and $37^{\circ} \mathrm{C}$ constant temperature conditions. The cell media were changed every one to two days. Some of the cells grown to the logarithmic phase were subcultured, and the other cells were frozen for future use.

3.1.6. Experimental Model. The human intestinal epithelial T84 cells were cultured in vitro to a confluent state, and different concentrations of urea $\left(0 \mathrm{mg} \mathrm{dL}^{-1}, 72 \mathrm{mg} \mathrm{dL}^{-1}\right.$, and $\left.144 \mathrm{mg} \mathrm{dL}^{-1}\right)$ and different durations $(24 \mathrm{~h}$ and $48 \mathrm{~h}$ ) were used to treat the T84 cells to simulate intestinal epithelial barrier injury. The effect of different concentrations of FSG on the viability of T84 cells was detected by the methyl thiazolyl tetrazolium (MTT) method, and the concentration of urea used for modeling was finally determined. 
3.1.7. Experimental Grouping. The cell treatment groups were as follows:

(1) Control group (group C): DMEM/F12 supplemented with $10 \%$ serum

(2) Model group (group M): DMEM/F12 supplemented with $10 \%$ serum and $144 \mathrm{mg} \mathrm{dL}^{-1}$ urea

(3) Low-dose FSG-containing serum group (FSG lowdose group): DMEM/F12 supplemented with $144 \mathrm{mg}$ $\mathrm{dL}^{-1}$ urea and $10 \%$ serum containing low-dose FSG

(4) High-dose FSG-containing serum group (FSG highdose group): DMEM/F12 supplemented with $144 \mathrm{mg}$ $\mathrm{dL}^{-1}$ urea and $10 \%$ serum containing high-dose FSG

\subsection{Detection}

3.2.1. MTT Assay to Detect the Effect of Different Concentrations of FSG on the Viability of T84 Cells. T84 cells were cultured and collected, followed by $0.25 \%$ trypsin digestion and Pekin dilution. The cell concentration was adjusted to 50,000 cells $\mathrm{mL}^{-1}$ and plated in 96-well plates. Every well contained $100 \mu \mathrm{L}$, with six double wells per group and incubated at $5 \% \mathrm{CO}_{2}$ at $37^{\circ} \mathrm{C}$. Until the cells grew to confluence, different concentrations of urea $\left(42 \mathrm{mg} \mathrm{dL}^{-1}, 72 \mathrm{mg}\right.$ $\mathrm{dL}^{-1}$, and $144 \mathrm{mg} \mathrm{dL}^{-1}$ ) and $10 \%$ serum containing low-dose FSG or high-dose FSG were added and further incubated in a $5 \% \mathrm{CO}_{2}$ environment at $37^{\circ} \mathrm{C}$ for $24 \mathrm{~h}$. MTT at $5 \mathrm{mg} \mathrm{mL}^{-1}$ or $0.5 \%$ was added to each well, followed by incubation at $37^{\circ} \mathrm{C}$ for another $4 \mathrm{~h}$ in a ventilated environment, and then, the supernatant was replaced with $150 \mu \mathrm{L}$ of dimethyl sulfoxide (DMSO) to fully dissolve the crystals with low-speed shaking for $10 \mathrm{~min}$. The absorbance was determined at $490 \mathrm{~nm}$ with an enzyme-linked immunoassay detector. Cell viability was calculated by the following formula: cell viability of control $=$ (medicine group A value - zero hole A value $) /($ control well A value - zero hole A value) $\times 100 \%$.

3.2.2. Cell Immunofluorescence Staining to Detect the Expression and Distribution of ZO-1 and Claudin-1. The logarithmic-phase cells were collected, digested with $0.25 \%$ pancreatin, diluted with culture medium, adjusted to a concentration of $1 \times 10^{6}$ cells on cell-climbing tablets in 24 well plates. Those cells, which were divided into the blank group, the model group (144 $\mathrm{mg} \mathrm{dL}^{-1}$ urea), the low-dose group of traditional Chinese medicine (FSG low-group), and the high-dose group of traditional Chinese medicine (FSG high-group), were treated with medicine and incubated at $37^{\circ} \mathrm{C}$ in $5 \% \mathrm{CO} 2$ until the cells grew to fusion. After incubation at $37^{\circ} \mathrm{C}$ for $24 \mathrm{~h}$, the cells were fixed with $4 \%$ paraformaldehyde (PA) for 20-30 minutes at room temperature. Then, the cells were washed with phosphate-buffered saline (PBS) twice for 5 minutes and treated with $0.5 \%$ Triton 100 for 10 minutes. After washing with PBS three times for $5 \mathrm{~min}$ at room temperature, the cells were incubated in $5 \%$ goat serum blocking solution at room temperature for $1 \mathrm{~h}$ and the cells were washed with PBS again. Rabbit anti-ZO-1 and claudin-1 (1:100 dilution) were added to the cells and incubated overnight at $4^{\circ} \mathrm{C}$.
The next day goat anti-rabbit IgG $(1: 200)$ was added and incubated in the dark at $37^{\circ} \mathrm{C}$ for $2 \mathrm{~h}$, after washing by PBS for $5 \mathrm{~min}$ three times. Then, the samples were washed again. 4',6-Diamidino-2-phenylindole (DAPI) antifluorescence quenching agent was added and incubated for $1 \mathrm{~min}$, and the slides were sealed and imaged under a laser confocal microscope.

3.2.3. Western Blots Were Used to Detect the Expression of ZO-1, Claudin-1, Nrf2, HO-1, MDA, and COX-2. T84 cells in the logarithmic growth stage were cultured synchronously and collected $48 \mathrm{~h}$ after treatment with $144 \mathrm{mg} \mathrm{dL}^{-1}$ urea and serum containing low and high dosages of FSG. The total protein was extracted and by lysing in RIPA buffer (containing 1\% proteinase inhibitor and phosphatase inhibitor). The protein concentration was determined by the BCA Protein Assay Kit (Boster, China). Protein $\left(10 \mu \mathrm{gmL}^{-1}\right)$ was separated on $10 \%$ sodium dodecyl sulfate (SDS)-polyacrylamide gel electrophoresis (PAGE), at $80 \mathrm{~V}$ constant voltage for $30 \mathrm{~min}, 140 \mathrm{~V}$ constant voltage for $40 \mathrm{~min}$, and a constant current of $200 \mathrm{~mA}$ for wet conversion for $2 \mathrm{~h}$; the proteins were transferred to a polyvinylidene fluoride (PVDF) membrane. After being transferred, the internal reference and target bands were cut according to the molecular weight of $200 \mathrm{~mA}$. After incubation, the corresponding antibody was incubated at $4^{\circ} \mathrm{C}$ overnight. After washing once with Tris-buffered saline and Tween (TBS-T) for $10 \mathrm{~min}$ three times, horseradish peroxidase (HRP)-labeled goat anti-rabbit or goat antimouse secondary antibody was added and incubated for $1 \mathrm{~h}$ at room temperature. After washing with TBST three times for $10 \mathrm{~min}$, enhanced chemiluminescence developer was added at a ratio of $1: 1$ to react for $1 \mathrm{~min}$, and the membrane was put it into a gel imaging system for development.

3.2.4. Statistical Analysis. Statistical analysis was performed by SPSS software v25.0. Data were presented as mean\pm standard error (SE). Multiple comparisons were done by one-way analysis of variance (ANOVA) followed by the Turkey multiple comparisons test. In the case of nonnormally distributed data, the Kruskal-Wallis test was used. A $P$ value $<0.05$ was considered statistically significant.

\section{Results}

4.1. Prediction of Traditional Chinese Medicine Targets and the Corresponding Active Ingredients. Each FSG component was input into the TCMSP for screening. Oral bioavailability $(\mathrm{OB} \geq 30 \%$ ) and drug similarity ( $\mathrm{DL} \geq 0.18$ ) were used as screening criteria. Finally, the screened target proteins were imported into UniProt and converted into gene names. A total of 2532 target genes were retrieved. Among them, 866 were associated with Danshen, 69 with Chenpi, 447 with Huangqi, 68 with Danggui, 511 with Yinyanghuo, 284 with Guijianyu, 173 with Banxia, and 114 with Dahuang. After removing the duplicates, a total of 378 genes were collected. 
4.2. Prediction of Targets Related to Chronic Renal Failure. The disease genes were searched in four databases: the NCBI (https://www.ncbi.nlm.nih.gov/), DisGeNET (https://www. disgenet.org/), Drugbank (https://www.drugbank.ca/), and the TTD (http://db.idrblab.net/ttd/) by entering "chronic renal failure" and limiting the species to humans. Finally, a total of 1327 genes were retrieved. Among them, NCBI retrieved a total of 63 genes, DisGeNET retrieved a total of 1251 genes, Drugbank retrieved a total of 16 genes, and TTD retrieved a total of one gene. After removing the duplicates, a total of 666 genes were collected.

4.3. Acquisition of Intersecting Genes. The potential gene targets of CRF-related genes and FSG active ingredients were used to draw a Venn diagram (http://bioinformatics.psb. ugent.be/webtools/Venn/) and the potential target genes in which FSG played an important role in CRF were obtained. The Venn diagram showed a total of 86 overlapping genes (Figure 1).

4.4. Construction and Analysis of PPINetwork. Since proteins often form macromolecular complexes through interactions, they can complete biological functions in cells [26]. To further explore the mechanism of FSG in treating CRF, we submitted the intersecting genes to STRING software to construct a PPI network and screened out target protein interaction data with confidence scores of $>0.9$. A total of 70 core target proteins were screened (Figure 2). Then, we applied Cytotype MCODE v 1.4.1 for further analysis, and the results showed that 17 central nodes were identified among the 70 nodes (Figure 3 ). Then, the intersecting genes were imported into Cytoscape software, the parameters were adjusted, and the results are shown in Figure 3. The network consisted of 174 nodes and 600 edges. In addition, we screened nine core genes based on the degree (Table 1). These genes are involved in various pathogenic CRF processes, including inflammation and fibrosis.

4.5. GO and KEGG Pathway Analysis of Target Proteins. To further clarify the biological role of these genes, GO function analysis of the intersecting genes was conducted in the DAVID database (https://david.ncifcrf.gov/) with significance indicated by a $P$ value of $<0.01$. The results showed the following: (1) for $\mathrm{BP}$, position regulation of transcription from the RNA polymer II promoter, informational response, and negative regulation of gene expression were identified; (2) for $\mathrm{MF}$, the intersecting genes were mainly enriched in the negative regulation of gene expression, heme binding, and sequence-specific DNA binding; (3) for the CCs, the 5 intersecting genes were mainly enriched in extractor exosomes, cytosol, and Golgi apparatuses (Table 2 and Figure 4).

To understand the action pathway of the 86 selected intersecting genes, KEGG pathway enrichment was analyzed in the DAVID database. KEGG enrichment was measured by gene number, $P$ value $(P<0.01)$, and enrichment factor. The results showed that these genes were significantly

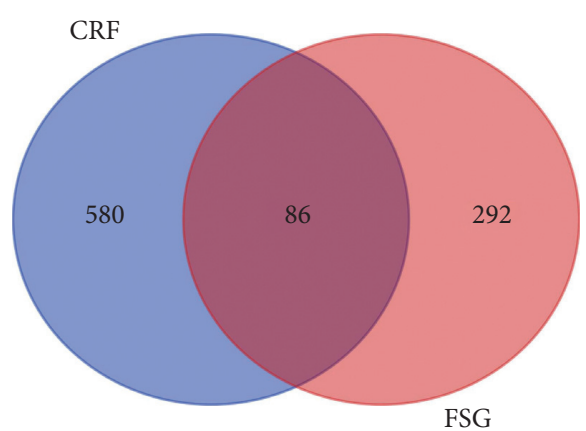

FIGURE 1: Eighty-six targets are common to FSG and CRF.

enriched in 61 pathways related to cancer, proteoglycans in cancer, the PI3K-Akt signaling pathway, and others. Here, we list only the top 20 pathways (Table 3 and Figure 5).

\subsection{Experimental Verification}

4.6.1. FSG Has a Nontoxic Effect on Uremic Toxin-Induced T84 Cells. The MTT experiment showed that treatment with $42 \mathrm{mg} \mathrm{dL}^{-1}, 72 \mathrm{mg} \mathrm{L}^{-1}$, and $144 \mathrm{mg} \mathrm{L}^{-1}$ urotoxin did not affect the viability of T84 cells $(P>0.05)$. Therefore, we selected $144 \mathrm{mg} \mathrm{L}^{-1}$ urotoxin for modeling. The cells were treated with low-dose FSG-containing serum and high-dose FSG-containing serum along with various concentrations of uremic toxin. High-dose FSG had a slight proliferative effect on cells in each urea treatment group, but with no statistical significance $(P>0.05$; Tables 4 and 5 and Figure 6).

4.6.2. Effect of FSG on the Expression of ZO-1 and Claudin-1 in Urotoxin-Treated T84 Cells. Claudin plays an important role in maintaining normal intestinal barrier function. ZO-1 and claudin-1 are two important tight junction proteins [29]. Previous studies have shown that, in animal models and human colon cells cultured in vitro, CKD induced a loss of intestinal epithelial tight junction proteins ZO-1 claudin-1 and occludin [6]. To observe the changes in ZO-1 and claudin-1 better, we performed using immunofluorescence staining assays to detect ZO- 1 and claudin- 1 in the T84 cells of each group. Compared to the blank group, the expression of ZO-1 (red) (Figure 7) and claudin-1 (red) (Figure 8) in the model group decreased, and the expression of ZO-1 and claudin-1 after treatment with low- and high-dose FSGcontaining serum increased. According to the results, the model was established successfully. The western blotting results showed compared to the control group, the expression levels of ZO-1 and claudin-1 in the model group decreased $(P<0.01)$. Compared to the model group, the expression levels of ZO-1 and claudin-1 in the low-dose group increased significantly $(P<0.01)$, and the expression levels of ZO-1 and claudin-1 in the high-dose group increased $(P<0.05)$ (Figure 9$)$.

4.6.3. Effect of FSG on Nrf2, HO-1, MDA, and COX-2 of T84 Cells. As shown in Figure 10, compared to the control group, the heme oxygenase-1 (HO-1) protein content 


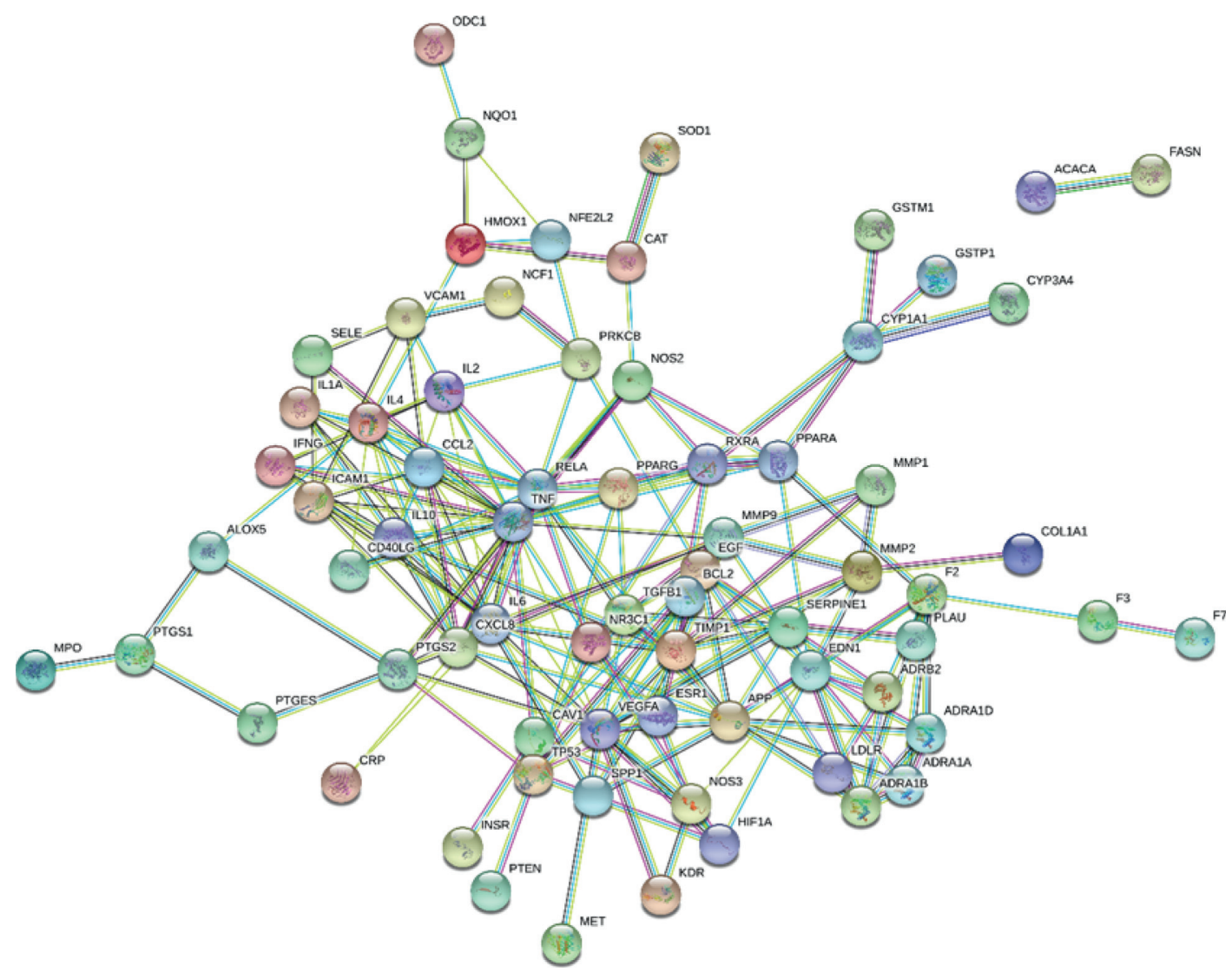

FIGURE 2: PPI network built by the STRING online database and module analysis.

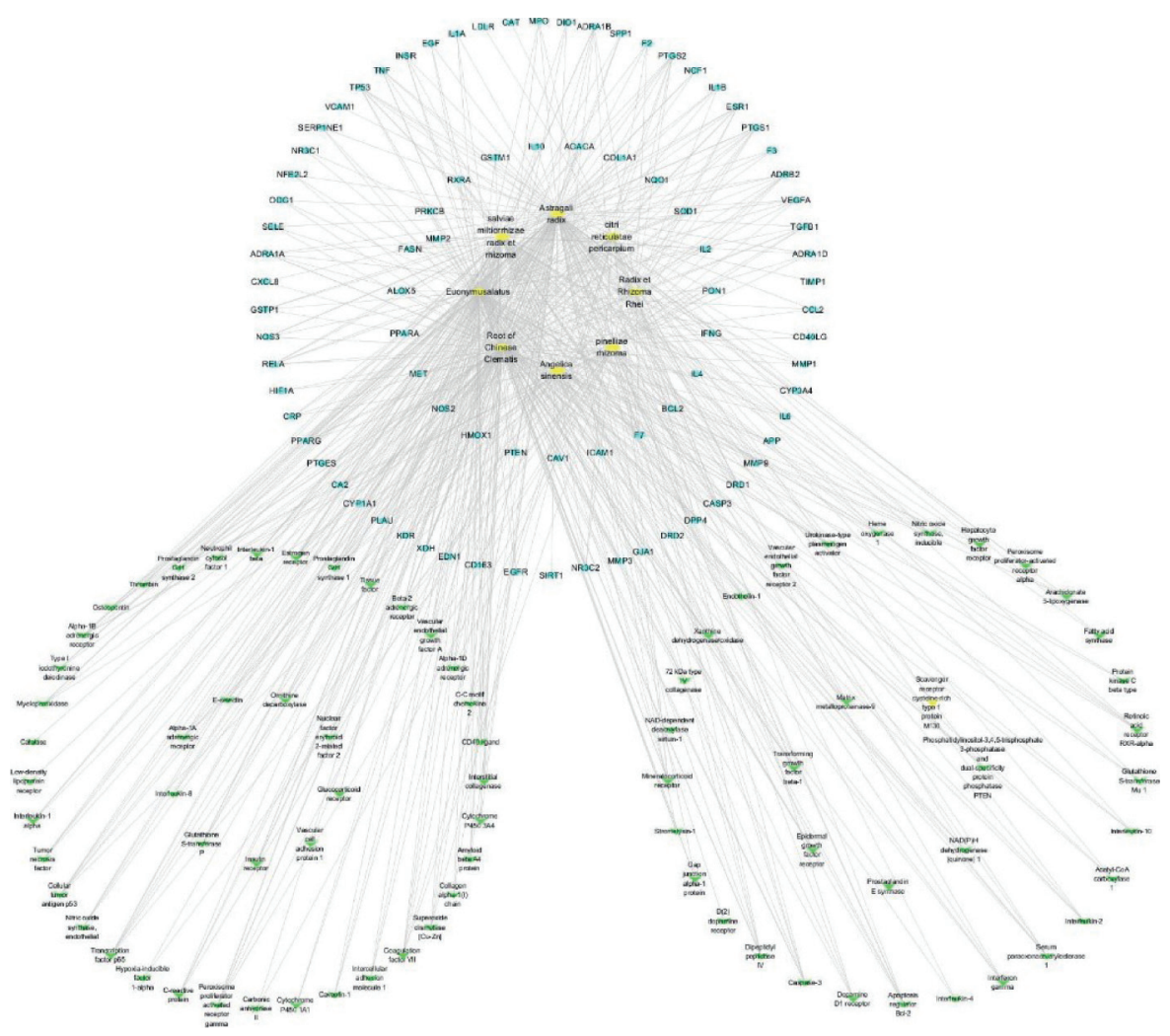

FiguRE 3: Drug-compound-target network. Yellow represents the drugs, blue represents the target genes, green represents the compounds, and the edge line represents the relationship between them. 
TABLE 1: Screening of core candidates.

\begin{tabular}{lccccccc}
\hline Name & $\begin{array}{c}\text { Average shortest } \\
\text { path length }\end{array}$ & Closeness centrality & Clustering coefficient & Stress & Degree & Betweenness centrality & Topological coefficient \\
\hline PTGS2 & 1.95402299 & 0 & 0.511765 & 16918 & 105 & 0.004564 & 0.464608 \\
PTGS1 & 1.95402299 & 0 & 0.511765 & 16918 & 68 & 0.004564 & 0.464608 \\
ADRB2 & 2.04597701 & 0 & 0.488764 & 9234 & 50 & 0.001962 & 0.51752 \\
DPP4 & 1.96551724 & 0 & 0.508772 & 15470 & 45 & 0.003893 & 0.512909 \\
RXRA & 2.06896552 & 0 & 0.483333 & 6292 & 40 & 0.001063 & 0.649057 \\
ADRA1B & 2.04597701 & 0 & 0.488764 & 9234 & 38 & 0.001962 & 0.51752 \\
PPARG & 1.96551724 & 0 & 0.508772 & 15470 & 37 & 0.003893 & 0.512909 \\
ESR1 & 1.96551724 & 0 & 0.508772 & 15470 & 35 & 0.003893 & 0.512909 \\
F2 & 2.11494253 & 0 & 0.472826 & 4998 & 34 & 0.001416 & 0.461538 \\
\hline
\end{tabular}

TABLE 2: GO analysis of candidate targets for FSG against CRF.

\begin{tabular}{|c|c|c|c|c|c|}
\hline Category & Term & Count & $\%$ & $P$ value & FDR \\
\hline GOTERM_BP_DIRECT & $\begin{array}{c}\text { GO: } 0045944 \sim \text { positive regulation of transcription from RNA } \\
\text { polymerase II promoter }\end{array}$ & 15 & 0.116505 & $7.96 E-07$ & 0.001275 \\
\hline GOTERM_BP_DIRECT & GO: $0006954 \sim$ inflammatory response & 9 & 0.069903 & $3.04 E-05$ & 0.048613 \\
\hline GOTERM_BP_DIRECT & GO: $0010629 \sim$ negative regulation of gene expression & 8 & 0.062136 & $1.68 E-07$ & $2.70 E-04$ \\
\hline GOTERM_BP_DIRECT & GO: $0001666 \sim$ response to hypoxia & 7 & 0.054369 & $1.51 E-06$ & 0.002417 \\
\hline GOTERM_BP_DIRECT & GO: $0045766 \sim$ positive regulation of angiogenesis & 7 & 0.054369 & $4.47 E-06$ & 0.007153 \\
\hline GOTERM_BP_DIRECT & GO: $0043066 \sim$ negative regulation of apoptotic process & 7 & 0.054369 & $1.83 E-03$ & 2.882482 \\
\hline GOTERM_BP_DIRECT & GO: $0006351 \sim$ transcription, DNA-templated & 7 & 0.054369 & $1.21 E-02$ & 17.70194 \\
\hline GOTERM_BP_DIRECT & GO: $0006979 \sim$ response to oxidative stress & 6 & 0.046602 & $2.45 E-05$ & 0.03918 \\
\hline GOTERM_BP_DIRECT & GO: $0001525 \sim$ angiogenesis & 6 & 0.046602 & $3.80 E-04$ & 0.605925 \\
\hline GOTERM_BP_DIRECT & GO: $0010628 \sim$ positive regulation of gene expression & 6 & 0.046602 & $8.81 E-04$ & 1.40093 \\
\hline GOTERM_MF_DIRECT & GO: $0005125 \sim$ cytokine activity & 11 & 0.085437 & $6.08 E-09$ & $7.57 E-06$ \\
\hline GOTERM_MF_DIRECT & GO: $0020037 \sim$ heme binding & 8 & 0.062136 & $7.12 E-06$ & 0.008875 \\
\hline GOTERM_MF_DIRECT & GO: $0043565 \sim$ sequence-specific DNA binding & 8 & 0.062136 & $2.47 E-03$ & 3.030466 \\
\hline GOTERM_MF_DIRECT & GO: $0008083 \sim$ growth factor activity & 7 & 0.054369 & $1.00 E-06$ & 0.001248 \\
\hline GOTERM_MF_DIRECT & GO: $0004252 \sim$ serine-type endopeptidase activity & 6 & 0.046602 & $1.17 E-03$ & 1.447175 \\
\hline GOTERM_MF_DIRECT & GO: $0042802 \sim$ identical protein binding & 5 & 0.038835 & $1.21 E-04$ & 0.151175 \\
\hline GOTERM_MF_DIRECT & GO: $0002020 \sim$ protease binding & 4 & 0.031068 & $4.22 E-04$ & 0.524268 \\
\hline GOTERM_MF_DIRECT & GO: $0003707 \sim$ steroid hormone receptor activity & 4 & 0.031068 & $4.95 E-03$ & 6.000497 \\
\hline GOTERM_MF_DIRECT & GO: $0042803 \sim$ protein homodimerization activity & 4 & 0.031068 & $1.19 E-02$ & 13.83665 \\
\hline GOTERM_MF_DIRECT & GO: $0004937 \sim$ alpha1-adrenergic receptor activity & 3 & 0.023301 & $1.36 E-04$ & 0.169113 \\
\hline GOTERM_CC_DIRECT & Extracellular space & 26 & 0.201942 & $3.25 E-13$ & $3.77 E-10$ \\
\hline GOTERM_CC_DIRECT & GO: $0070062 \sim$ extracellular exosome & 18 & 0.139806 & $7.97 E-03$ & 8.860488 \\
\hline GOTERM_CC_DIRECT & GO: $0005829 \sim$ cytosol & 16 & 0.124272 & $1.18 E-05$ & 0.013679 \\
\hline GOTERM_CC_DIRECT & GO: $0005794 \sim$ Golgi apparatus & 9 & 0.069903 & $1.16 E-03$ & 1.340888 \\
\hline GOTERM_CC_DIRECT & GO: $0005576 \sim$ extracellular region & 9 & 0.069903 & $2.31 E-03$ & 2.648495 \\
\hline GOTERM_CC_DIRECT & GO: $0045121 \sim$ membrane raft & 7 & 0.054369 & $6.90 E-06$ & 0.007999 \\
\hline GOTERM_CC_DIRECT & GO: $0009897 \sim$ external side of plasma membrane & 7 & 0.054369 & $6.50 E-05$ & 0.075398 \\
\hline GOTERM_CC_DIRECT & GO: $0009986 \sim$ cell surface & 7 & 0.054369 & $3.62 E-03$ & 4.113532 \\
\hline GOTERM_CC_DIRECT & GO: $0031012 \sim$ extracellular matrix & 6 & 0.046602 & $1.67 E-04$ & 0.19341 \\
\hline GOTERM_CC_DIRECT & GO: $0005925 \sim$ focal adhesion & 6 & 0.046602 & $1.08 E-02$ & 11.87756 \\
\hline
\end{tabular}

increased $(P<0.01)$, the Nrf2 total protein content decreased $(P<0.01)$, and Nrf2 and HO-1 protein expression in the group treated with high- and low-dose serum containing FSG increased $(P<0.01)$. Compared to the blank group, the expression level of cyclooxygenase-2 (COX-2) protein and MDA protein in the model group was significantly higher $(P<0.01)$. The expression level of MDA in the groups treated with serum containing FSG at each concentration was increased significantly $(P<0.01)$, and the differences were statistically significant. The expression level of COX-2 in the groups treated with serum containing FSG at each concentration was lower $(P<0.05)$.

\section{Discussion}

5.1. Network Pharmacology and FSG. This study systematically explored the pharmacological mechanism of FSG in the treatment of gastrointestinal dysfunction in chronic renal failure through network pharmacological analysis and experimental verification. Because traditional Chinese medicine has a lot of medicinal exerts and complex ingredients, the effective ingredients of many drugs are not clear at present. Thus, it is difficult for traditional research methods to clarify their mechanisms. We analyzed the compound composition of each drug in FSG through network pharmacology and then used these compounds to identify the 


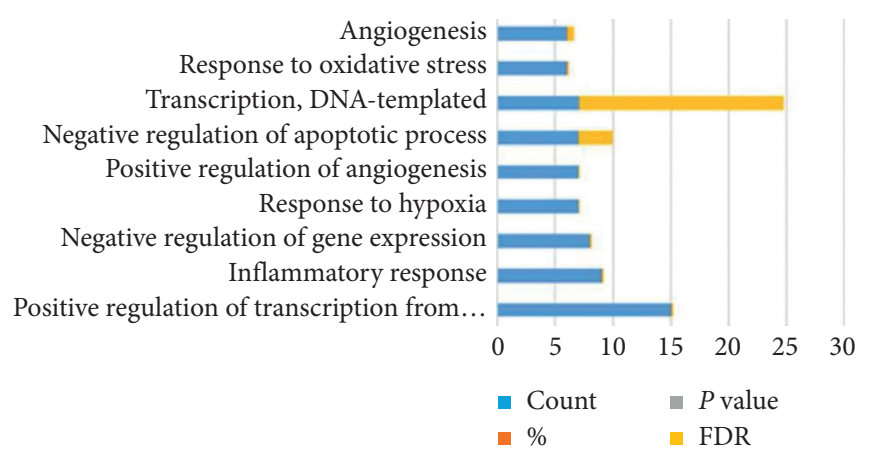

(a)

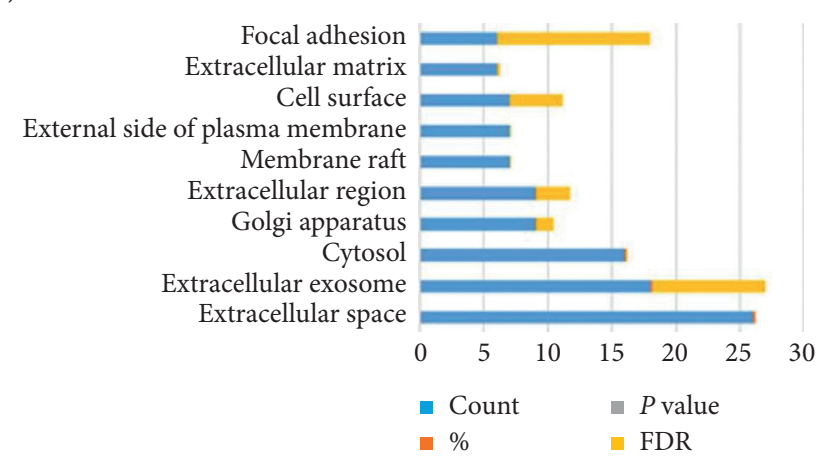

(c)

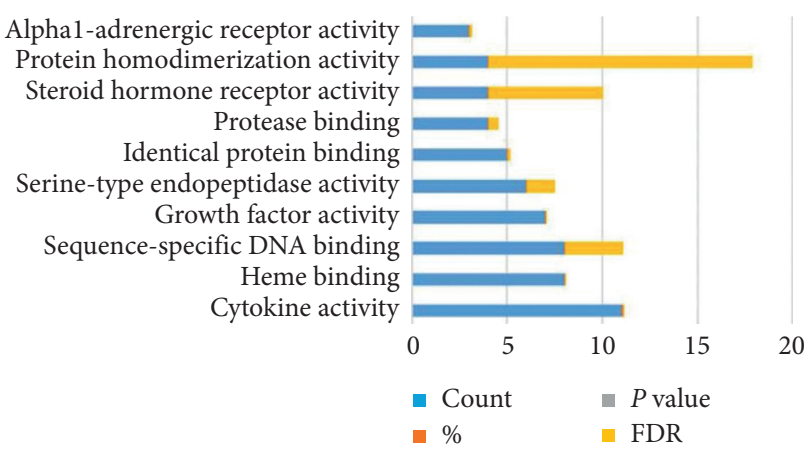

(b) (b) Analysis of 10 molecular functions of the core targets of the effect of FSG on CRF. (c) Analysis of 10 cell components of the core target of the effect of FSG on CRF.

corresponding target proteins and gene names. The same method was used to find the intersection of target proteins and genes corresponding to CRF, and 86 potential protein targets for treating CRF with FSG were analyzed. Then through the STRING network, we obtained the protein interaction network map.

Cytoscape was used to construct the FSG Chinese medicine-compound-target network diagram, which had 174 nodes and 600 edges. Among the 174 nodes, there were 81 compound nodes, 85 target nodes, and 8 drug nodes. The results showed that FSG had multicomponent, multitarget, and synergistic treatment characteristics. Then, we applied network analysis to screen out 9 core candidates. We determined the 9 core candidate components of FSG in treating CRF according to the degree. PTGS2 (prostaglandin G/H synthase 2), PTGS1 (prostaglandin $\mathrm{G} / \mathrm{H}$ synthase 1 ), ADRB2 (beta-2 adrenergic receptor), DPP4 (dipeptidyl peptidase IV), RXRA (retinoic acid receptor RXR-alpha), ADRA1B (alpha-1B adrenergic receptor), PPARG (peroxisome proliferator-activated receptor-gamma), ESR1 (estrogen receptor), and F2 (thrombin) were closely related to the active components of FSG in treating CRF. Among them, PTGS2, also known as COX-2, had the highest intermediate level. COX-2 has very low activity in normal tissue cells, while in inflammatory cells, cytokines and mitogenic factors can significantly induce COX-2 expression [30]. Studies have shown that COX-2 plays an important role in the regulation of various chronic kidney diseases [31]. At the same time, COX-2 plays a key role in regulating renin release and water and salt metabolism and maintaining blood pressure $[32,33]$. They interact with each other to delay the progression of CRF through different signaling pathways.

To clarify how FSG worked through these targets, this study further analyzed GO and KEGG pathway enrichment. FSG was found to play anti-inflammatory and antioxidation roles by regulating the activity of some cytokines and growth factors, and FSG also plays an important part in regulating the negative regulation of apoptosis, angiogenesis, RNA regulation, and coping with hypoxia. Signal transmission is mainly on the surface of the cell membrane, Golgi, extracellular matrix, and the outer side of the plasma membrane. The KEGG results showed that FSG may be involved in the cancer signaling pathway, the PI3K-Akt signaling pathway, the HIF1 signaling pathway, and the TNF signaling pathway. The PI3K/ Akt signaling pathway is involved in biological activities including apoptosis, metabolism, proliferation, and differentiation. Previous studies have proved that the PI3K/Akt signaling pathway plays an important role in the treatment of renal failure and hypertension [34]. However, the exact mechanism needs further study. Hypoxia-inducible factor 1 (HIF1) is an important factor for maintaining oxygen balance in the body. Under hypoxic conditions, the glomeruli, the capillary network around the renal tubules, and the blood vessels entering and exiting the small arteries contract, 
TABLE 3: Enrichment analysis of candidate targets for FSG against CRF.

\begin{tabular}{|c|c|c|c|c|c|}
\hline Category & Term & Count & $\%$ & $\begin{array}{c}P \\
\text { value }\end{array}$ & Genes \\
\hline KEGG_PATHWAY & ssc05200: pathways in cancer & 20 & 0.155339806 & 0.00 & $\begin{array}{l}\text { EGFR, IL6, PTGS2, RELA, MMP9, PPARG, MET, } \\
\text { TP53, CXCL8, PTEN, MMP2, TGFB1, MMP1, } \\
\text { PRKCB, CASP3, HIF1A, BCL2, VEGFA, NOS2, EGF }\end{array}$ \\
\hline KEGG_PATHWAY & $\begin{array}{l}\text { ssc05205: proteoglycans in } \\
\text { cancer }\end{array}$ & 15 & 0.116504854 & 0.00 & $\begin{array}{c}\text { EGFR, CAV1, TNF, MMP9, MET, TP53, ESR1, } \\
\text { MMP2, TGFB1, PRKCB, KDR, CASP3, HIF1A, } \\
\text { VEGFA, PLAU }\end{array}$ \\
\hline KEGG_PATHWAY & $\begin{array}{r}\text { ssc04151: PI3K- } \\
\text { pathw }\end{array}$ & 15 & 0.116504854 & 0.00 & $\begin{array}{l}\text { EGFR, IL4, IL6, RELA, MET, TP53, PTEN, KDR, } \\
\text { BCL2, VEGFA, NOS3, EGF, INSR, IL2, SPP1 }\end{array}$ \\
\hline KEGG_PATHWAY & $\begin{array}{l}\text { ssc04066: HIF-1 signaling } \\
\text { pathway }\end{array}$ & 14 & 0.108737864 & 0.00 & $\begin{array}{l}\text { EGFR, IL6, HIF1A, RELA, BCL2, } \\
\text { VEGFA, NOS3, NOS2, EGF, INSR, }\end{array}$ \\
\hline KEGG_PATHWAY & ssc05144: malaria & 12 & 0.093203883 & 0.00 & $\begin{array}{r}\text { VCAM1, ICAM1, IL6, CCL2, TNF, CD } \\
\text { MET, CXCL8, SELE, TGFB1, I }\end{array}$ \\
\hline KEGG_PATHWAY & $\begin{array}{r}\text { ssc04668: TN } \\
\text { pathy }\end{array}$ & 12 & 0.093203883 & 0.00 & $\begin{array}{r}\text { VCAM1, ICAM1, CASP3, IL6, } \\
\text { MMP9, RELA, EDN1, }\end{array}$ \\
\hline KEGG_PATHWAY & $\begin{array}{l}\text { ssc05206: micro-RNAs in } \\
\text { cancer }\end{array}$ & 12 & 0.093203883 & 0.00 & $\begin{array}{r}\text { EGFR, CASP3, PTGS2, BCL2, MI } \\
\text { TP53, PTEN, SIRT1, PL }\end{array}$ \\
\hline KEGG_PATHWAY & $\begin{array}{l}\text { ssc05323: rheumatoid } \\
\text { arthritis }\end{array}$ & 11 & 0.085436893 & 0.00 & $\begin{array}{r}\text { ICAM1, IL6, CCL2, TNF, IFNG, } \\
\text { MMP3, TGFB1, MMP }\end{array}$ \\
\hline KEGG_PATHWAY & ssc05145: & 1 & 0. & 0.00 & $\begin{array}{c}\text { CASP3, TNF, LDLR, CD40LG, BCL2, RELA, IFNG, } \\
\text { NOS2, ALOX5, TGFB1, IL10 }\end{array}$ \\
\hline KEGG_PATHWAY & 5805161. & 11 & 3 & 0.00 & $\begin{array}{l}\text { CASP3, IL6, TNF, BCL2, MMP9, RELA, TP53, } \\
\text { CXCL8, PTEN, TGFB1, PRKCB }\end{array}$ \\
\hline KEGG_PATHWAY & $\begin{array}{l}\text { ssc04060: cytokine-cytokine } \\
\text { receptor interaction }\end{array}$ & 1 & 0.085436893 & 0.00 & $\begin{array}{l}\text { IL4, IL6, CCL2, TNF, CD40LG, IFNG, CXCL8, } \\
\text { TGFB1, IL1A, IL10, IL2 }\end{array}$ \\
\hline KEGG_PATHWAY & ssc05140: leishmaniasis & 10 & 0.077669903 & 0.00 & $\begin{array}{c}\text { IL4, TNF, PTGS2, NCF1, RELA, IFNG, NOS2, } \\
\text { TGFB1, IL1A, IL10 }\end{array}$ \\
\hline KEGG_PATHWAY & ssc05146: & 1 & 7669903 & 0.00 & $\begin{array}{l}\text { CASP3, IL6, TNF, RELA, IFNG, CXCL8, NOS2, } \\
\text { TGFB1, IL10, PRKCB }\end{array}$ \\
\hline KEGG_PATHWAY & $\begin{aligned} \operatorname{ssc}( \\
(\mathrm{Ame}\end{aligned}$ & 10 & 0.077669903 & 0.00 & $\begin{array}{l}\text { IL6, CCL2, TNF, RELA, IFNG, CXCL8, NOS2, } \\
\text { TGFB1, IL10, IL2 }\end{array}$ \\
\hline KEGG_PATHWAY & $\begin{array}{l}\text { ssc05202: transcriptional } \\
\text { misregulation in cancer }\end{array}$ & 10 & 0.077669903 & 0.00 & $\begin{array}{c}\text { IL6, MMP9, RELA, MET, PPARG, TP53, CXCL8, } \\
\text { MPO, MMP3, PLAU }\end{array}$ \\
\hline KEGG_PATHWAY & ssc05152: tuberculosis & 10 & 0.077669903 & 0.00 & $\begin{array}{l}\text { CASP3, IL6, TNF, BCL2, RELA, IFNG, NOS2, } \\
\text { TGFB1, IL1A, IL10 }\end{array}$ \\
\hline KEGG_PATHWAY & ssc04510: focal adhesion & 10 & 0.077669903 & 0.00 & $\begin{array}{c}\text { EGFR, CAV1, BCL2, VEGFA, MET, EGF, PTEN, } \\
\text { PRKCB, KDR, SPP1 }\end{array}$ \\
\hline KEGG_PATHWAY & $\begin{aligned} \text { ssc05321: } \\
\text { di }\end{aligned}$ & 9 & 69902913 & 0.00 & IL4, IL6, TNF, RELA, IFNG, TGFB1, IL1A, IL10, IL2 \\
\hline KEGG_PATHWAY & $\begin{array}{l}\text { ssc04064: NF-kappa B } \\
\text { signaling pathway }\end{array}$ & 9 & 0.069902913 & 0.00 & $\begin{array}{l}\text { VCAM1, ICAM1, TNF, PTGS2, CD40LG, BCL2, } \\
\text { RELA, CXCL8, PLAU }\end{array}$ \\
\hline KEGG_PATHWA) & $\begin{array}{l}\text { ssc04068: FoxO signaling } \\
\text { pathway }\end{array}$ & 9 & 0.069902913 & 0.00 & $\begin{array}{l}\text { EGFR, IL6, CAT, EGF, INSR, PTEN, SIRT1, TGFB1, } \\
\text { IL10 }\end{array}$ \\
\hline
\end{tabular}

resulting in decreased blood flow. This decreases the glomerular filtration rate, and the tubule interstitial becomes hypoxic, which leads to renal fibrosis [30]. Therefore, chronic hypoxia and CRF are linked, and the role of FSG in these processes needs further study. Cancer signaling pathways such as the MAPK signaling pathway [35], NF- $\kappa \mathrm{B}$ signaling pathway [36], and Wnt signaling pathway [37] are all linked to chronic renal failure. Mitogen-activated protein kinase (MAPK) is a functionally related kinase that regulates key cellular processes (e.g., survival, death, differentiation, and proliferation) involved in kidney disease [38]. Nuclear factor$\mathrm{kB}(\mathrm{NF}-\mathrm{KB})$ is an important nuclear transcription factor in cells. It plays a key role in the regulation of genes such as fibronectin (FN), tumor necrosis factor- $\alpha$ (TNF- $\alpha$ ), interleukin-8 (IL-8), and monocyte chemoattractant protein-1 $(\mathrm{MCP}-1)$ related to inflammatory responses, thus aggravating renal damage [39]. The $\mathrm{Wnt} / \beta$ catenin signaling pathway is a classical Wnt pathway. The $\mathrm{Wnt} / \beta$ catenin signaling pathway was reported to have little activity in normal adult kidneys but was activated after various kidney injuries [40, 41]. Oxidative stress and inflammation also play important roles in cancer signaling pathways $[42,43]$. A previous GO enrichment analysis found that FSG had obvious anti-inflammatory and antioxidant effects in the treatment of CRF. Previous studies have shown that FSG could correct intestinal dysfunction in patients with peritoneal dialysis and protect residual renal 


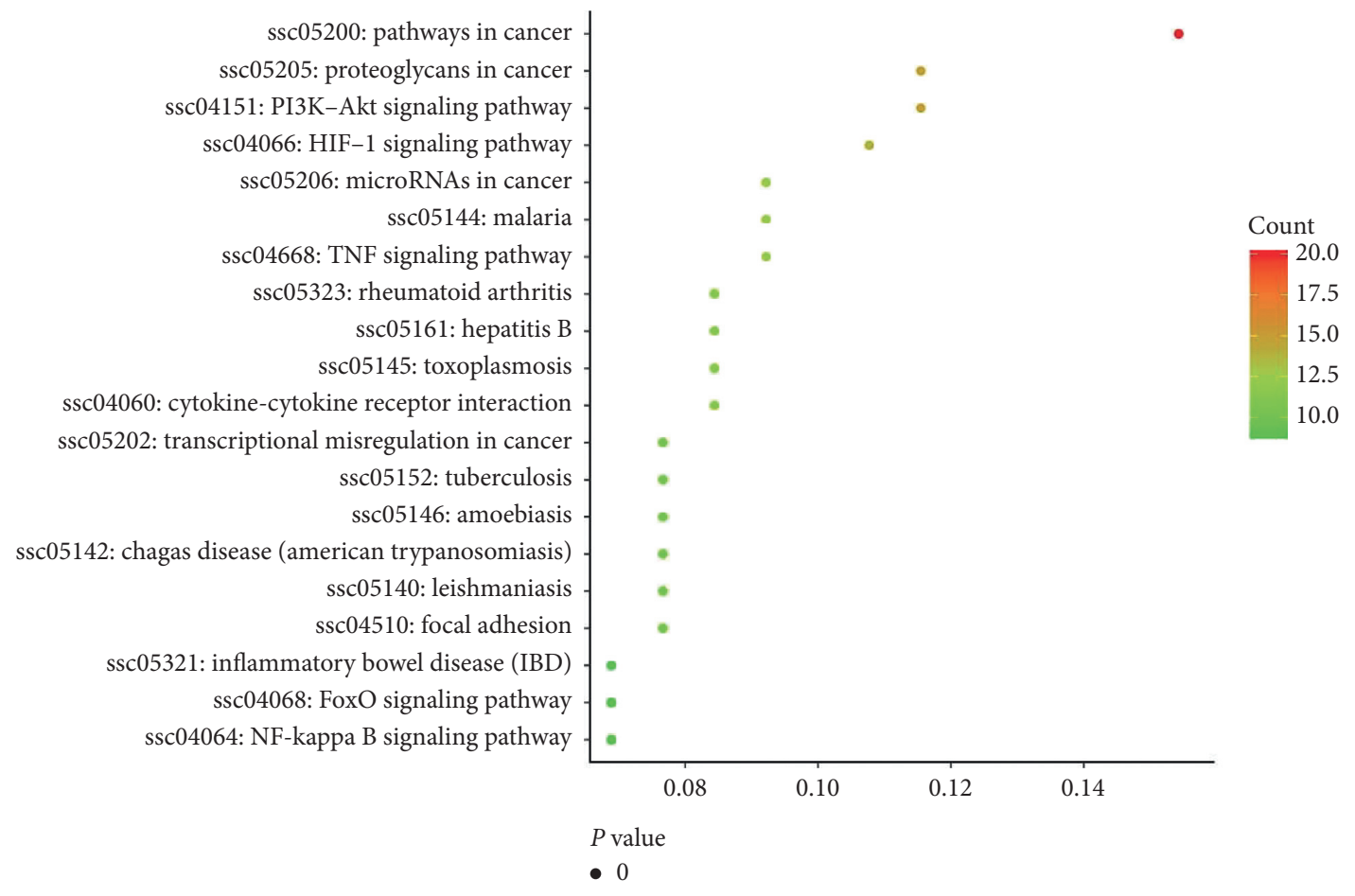

FIGURE 5: Enrichment analysis of candidate targets for FSG against CRF.

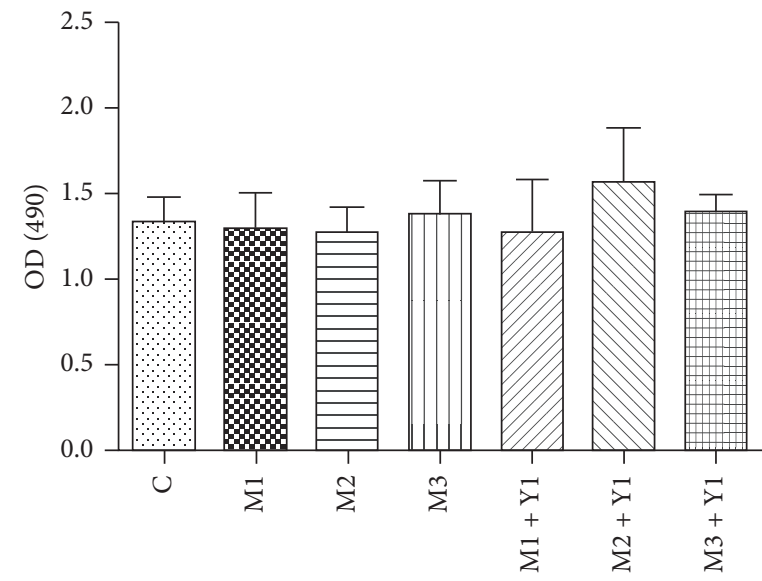

(a)

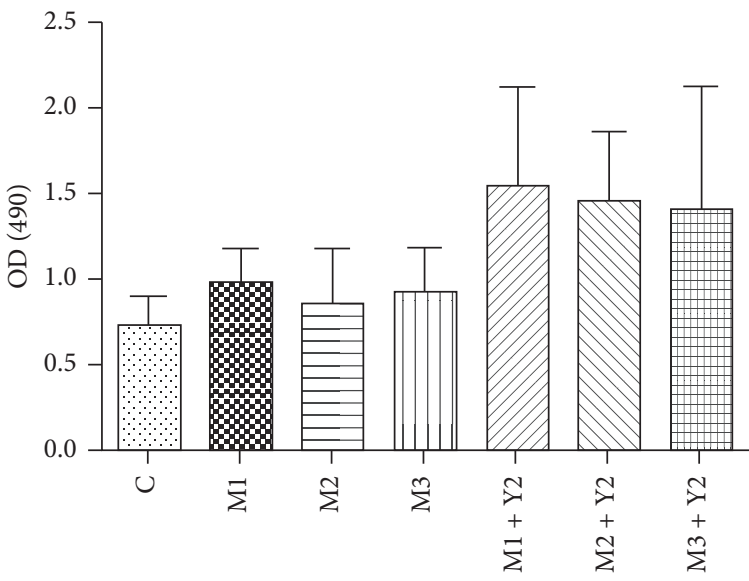

(b)

FIgURE 6: Effect of various concentrations of uremic toxin and low (a) and high-dose (b) FSG-containing serum on T84 viability.

function [44]. Therefore, through the network pharmacological analysis, experiments on oxidative stress and antiinflammation were conducted.

\subsection{Experimental Study of FSG on Chronic Renal Failure} Gastrointestinal Dysfunction. Gastrointestinal dysfunction in CRF is very common in patients with CRF and ESRD. The stimulation of the gastrointestinal tract by uremia toxins and some metabolites has long been considered to be an important cause of gastrointestinal dysfunction in CRF [45]. In this study, human colon epithelial cells (T84) were cultured in vitro to establish an intestinal barrier injury model of CRF induced by urotoxin. Changes in tight junction proteins $\mathrm{ZO}-1$ and claudin-1, inflammatory factor COX-2, and Nrf2/HO-1 signal transduction in intestinal epithelial cells were observed. The study found that serum containing different concentrations of FSG could upregulate the expression of intestinal epithelial tight junction proteins claudin-1 and ZO-1 to different degrees and played a protective role in the intestinal epithelial barrier of T84 cells. As an inducible enzyme, COX-2 can initiate and regulate the inflammatory response of tissues [46]. This study found that the COX-2 protein in the model group was significantly increased, whereas the COX-2 protein 


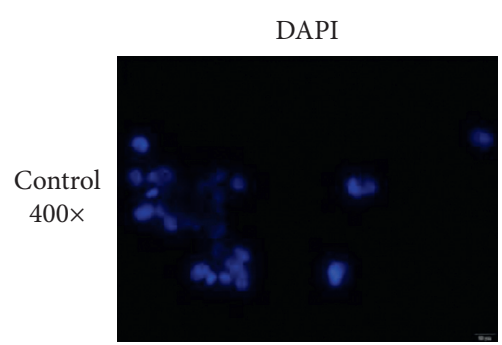

(a)

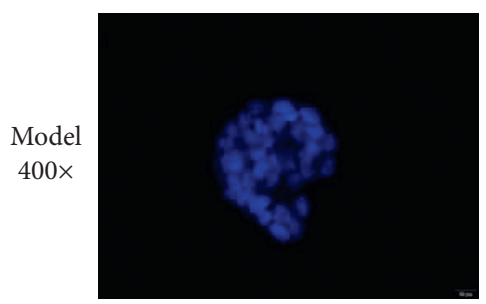

(d)

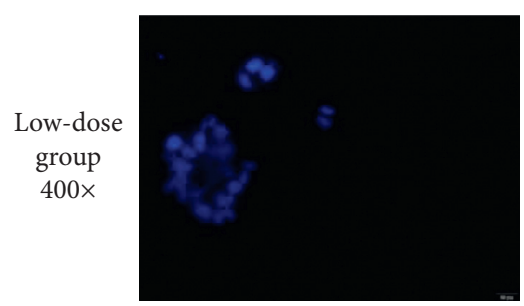

(g)

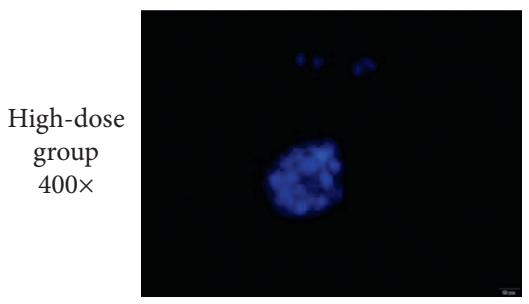

(j)

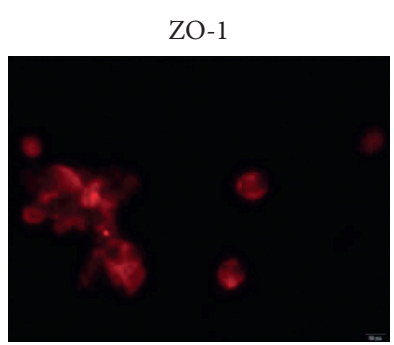

(b)

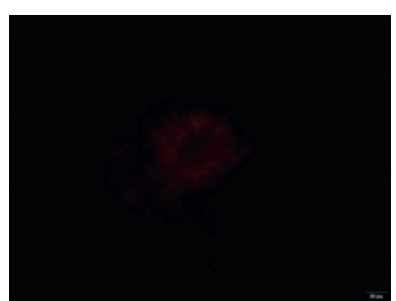

(e)

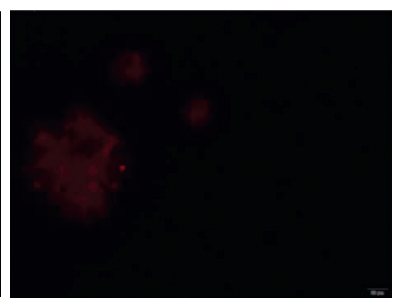

(h)

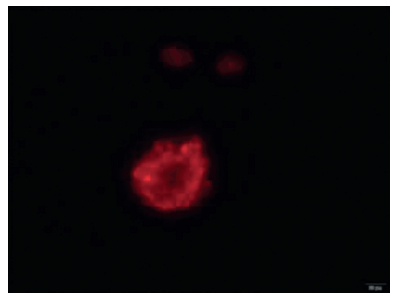

(k)

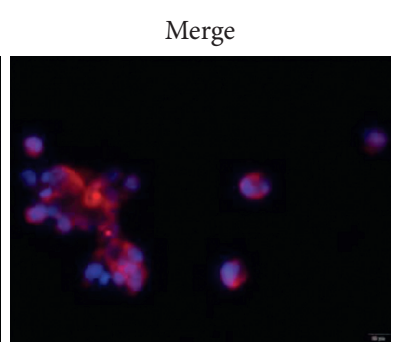

(c)

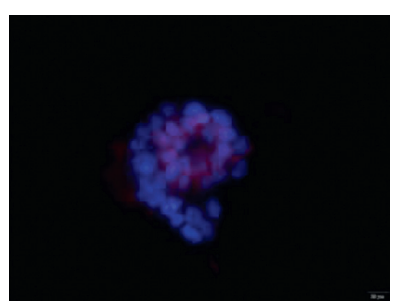

(f)

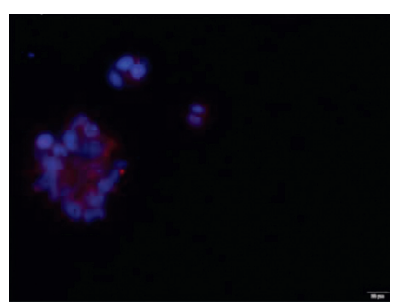

(i)

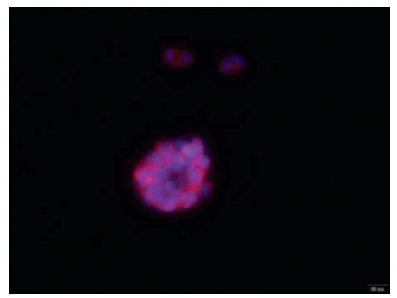

(1)

Figure 7: The protein levels of ZO-1 in T84 cells based on immunofluorescence staining.

in each FSG treatment group was decreased $(P<0.05)$. FSG has been suggested to improve intestinal epithelial barrier cells injured by urotoxin by inhibiting inflammatory reactions. Nrf2 is the most important transcriptional regulator of oxidative stress and inflammation. A large amount of evidence indicates that Nrf2 protects cells from oxidative stress by activating the expression of genes encoding detoxification, antioxidant, and anti-inflammatory proteins [47]. Under physiological conditions, Nrf2 is isolated in the cytoplasm by Kelch-like ECH-related protein 1 (Keap1). However, when organisms are exposed to oxidative stress or some toxic effects (such as ultraviolet radiation, radiation, and air pollution), Nrf2 is dissociated from Keap1, rapidly translocates into the nucleus, combines with MAF protein to form a dimer, and then combines with the antioxidant response element (ARE), regulating the expression of 65 antioxidant genes, including NADPH quinone oxidoreductase 1 (NQO1) and HO-1 [48-50]. This study showed that FSG could increase the expression of Nrf2 and HO-1. FSG has been suggested to improve the intestinal barrier injury in CRF by mediating the Nrf2/HO-1 signaling pathway. MDA is an important marker of oxidative stress. Under stress conditions, the generation of oxygen-free radicals in the body increases, and oxygen-free radicals participate in the oxidative stress reaction and eventually generate the oxidative end-product MDA [51], as found in the present study. This study found that FSG could reduce the inflammatory state of $\mathrm{T} 84$ cells caused by urotoxin by reducing the COX-2 protein content. At the same time, FSG upregulated the expression of $\mathrm{Nrf} 2$ and $\mathrm{HO}-1$, cleared the excessive accumulation of MDA in T84 cells, enhanced the cell antioxidant defense capability, and alleviated the effect of urotoxin on the oxidative stress of T84 cells. This study was the first to use network pharmacology to study the mechanism of FSG in treating CRF and conduct further in vitro experiments. The traditional Chinese medicine FSG reduced the inflammatory state of T84 cells induced by urotoxin through the Nrf2/HO-1 signaling pathway and decreased the damage to intestinal barrier function in CRF. This study had 


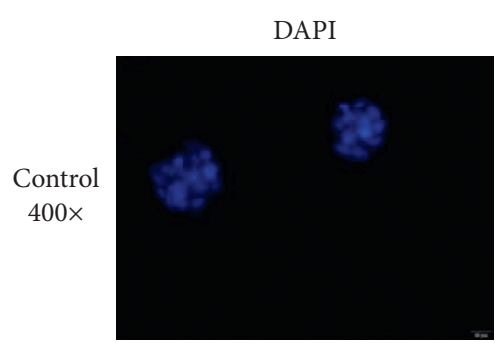

(a)

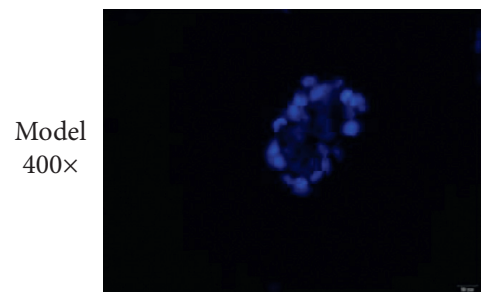

(d)

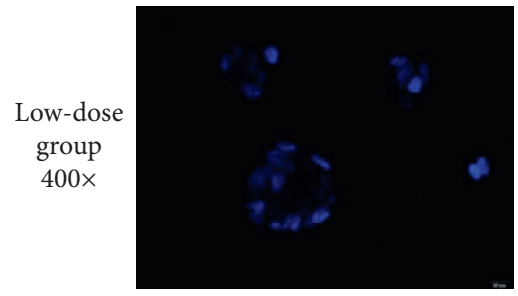

(g)

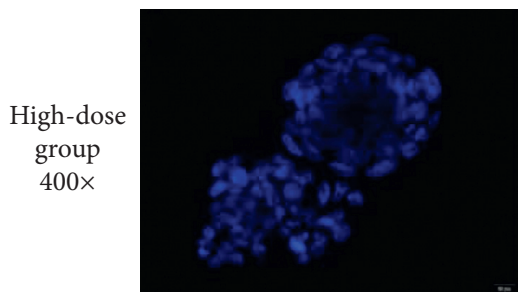

(j)
Claudin-1

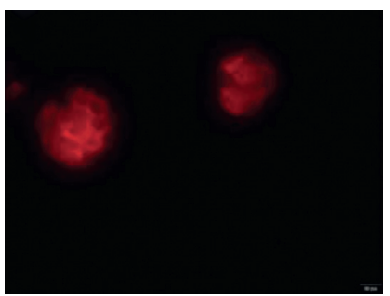

(b)

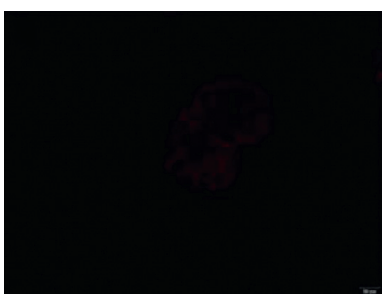

(e)

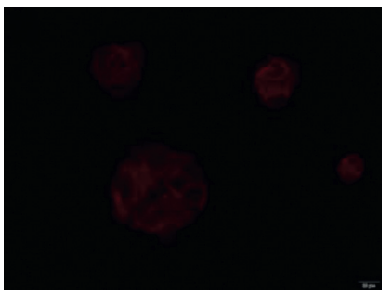

(h)

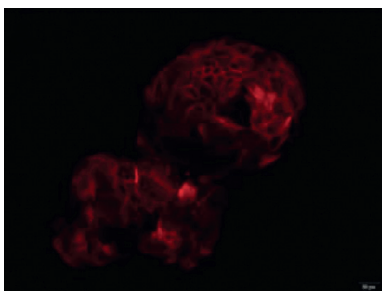

(k)

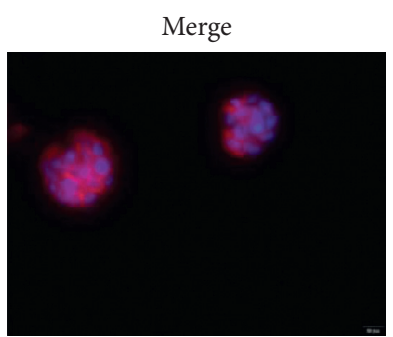

(c)

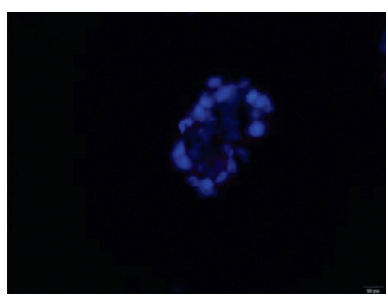

(f)

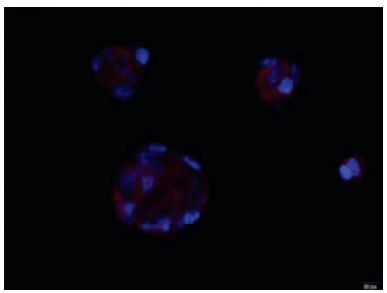

(i)

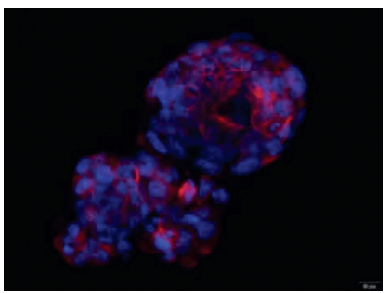

(l)

FIGURE 8: The protein levels of claudin-1 in T84 cells based on immunofluorescence staining.
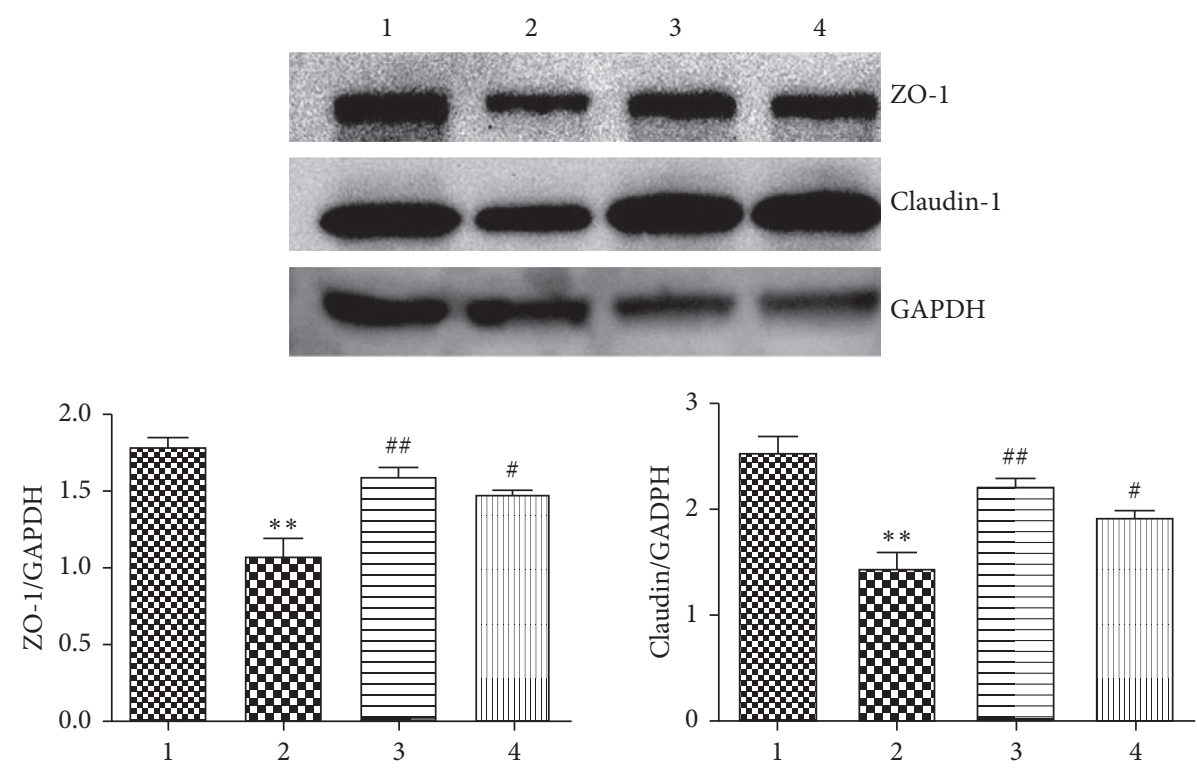

FIGURE 9: Protein expression of ZO-1 and claudin-1. The data are expressed as the mean \pm SE, $n=4$. 1: group C; 2 : group M; 3: FSG low-dose group; 4 : FSG high-dose group. Compared to the control group, ${ }^{*} P<0.05$ and ${ }^{*}{ }^{*} P<0.01$; compared to the model group, ${ }^{\#} P<0.05$ and ${ }^{\# \#} P<0.01$. 


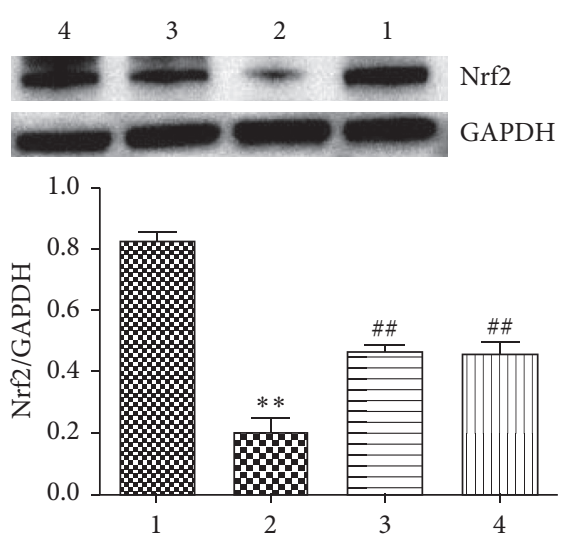

(a)

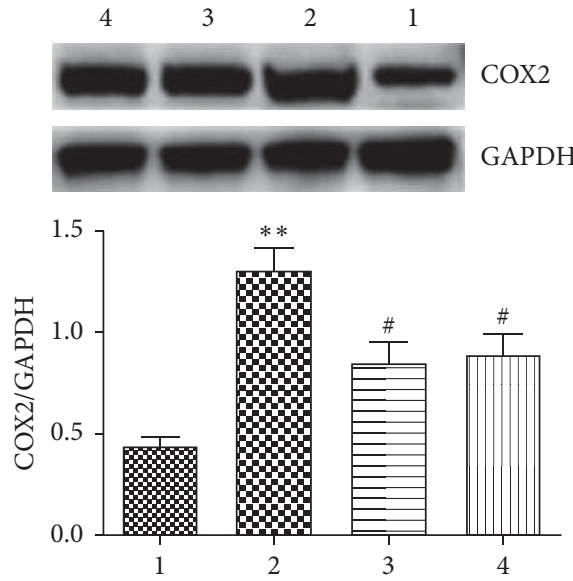

(c)

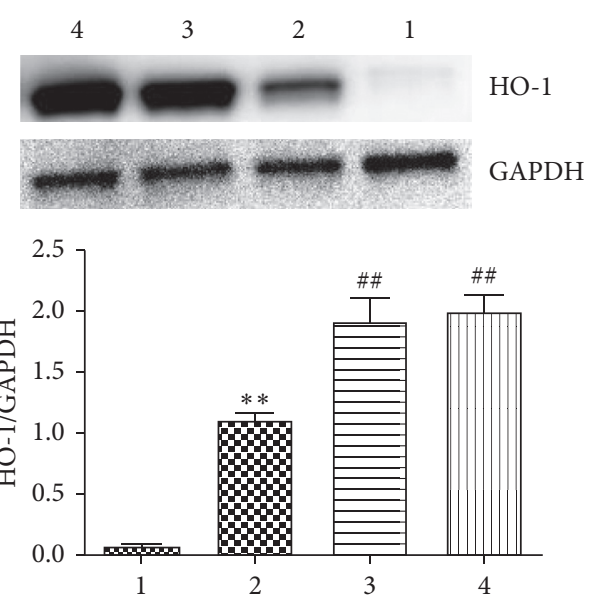

(b)
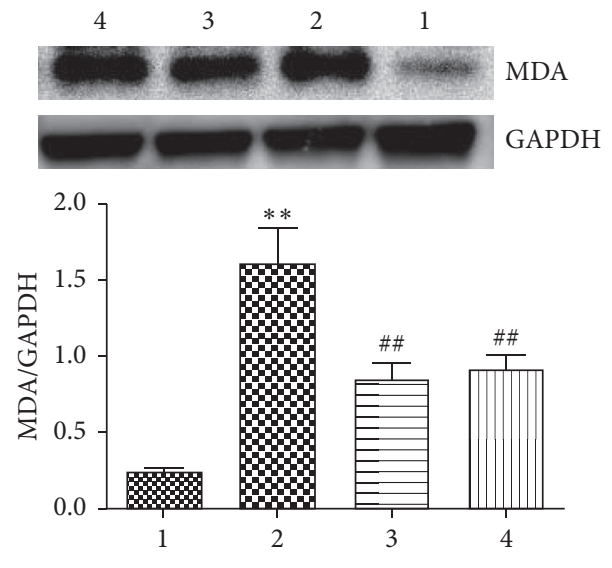

(d)

FIGURE 10: The effect of FSG on T84 intestinal epithelial cells: (a) protein expression of Nrf2; (b) protein expression of HO-1; (c) protein expression of COX-2; (d) protein expression of MDA. The data are expressed as the mean \pm SE, $n=4$; 1 , group C; 2 , group M; 3 , FSG lowdose group; 4, FSG high-dose group. Compared to the control group, ${ }^{*} P<0.05$ and ${ }^{*}{ }^{*} P<0.01$; compared to the model group, ${ }^{\#} P<0.05$ and ${ }^{\# \#} P<0.01$.

several shortcomings. Firstly, due to the limitations of the experimental conditions, only in vitro experiments were conducted, not in vivo experiments. Secondly, we did not detect the regulatory effect of urotoxin and FSG on Nrf2 protein expression in T84 cells. However, we did find that FSG upregulates the total Nrf2 protein expression. Therefore, the regulatory effect of FSG on HO-1 may be related to the promotion of Nrf2 into the nucleus. We will verify this hypothesis in a follow-up study.

\section{Conclusion}

FSG has a protective effect on urinary toxin-induced intestinal epithelial barrier injury in CRF, and its mechanism may be related to its upregulation of $\mathrm{Nrf} 2 / \mathrm{HO}-1$ signal transduction and thus the inhibition of tissue oxidative stress and inflammatory responses. Screening CRF targets and identifying the corresponding FSG components using a network pharmacological method is a practical strategy to explore the mechanism of FSG in improving gastrointestinal dysfunction in CRF.

\author{
Abbreviations \\ CKD: Chronic kidney disease \\ FSG: $\quad$ Fushen Granule \\ CRF: Chronic renal failure \\ ZO-1: Zonula occludens-1 \\ Nrf2: $\quad$ Nuclear factor E2-related factor 2 \\ HO-1: Heme oxygenase 1 \\ MDA: Malondialdehyde \\ COX-2: Cyclooxygenase-2 \\ TCMSP: Chinese medicine system pharmacology database \\ and analysis platform \\ GO: $\quad$ Gene ontology \\ KEGG: Kyoto encyclopedia of genes and genomes \\ pathway.
}

\section{Data Availability}

All data generated or analyzed during this study are available from the corresponding author upon request. 


\section{Disclosure}

The funders had no role in the study design, data collection and analysis, decision to publish, or preparation of the manuscript.

\section{Conflicts of Interest}

The authors declare that there are no conflicts of interest.

\section{Authors' Contributions}

MRH and HXY designed the study. MRH, HXY, and PYL participated in editing the manuscript and performed the experiments. MRH and HFY analyzed the data and wrote the manuscript. ZHY and KY helped with data collection. $\mathrm{MRH}$ and $\mathrm{KY}$ were responsible for funding acquisition, project administration, and validation of this article. All the authors read and approved the final manuscript. Miaoru Han and Hangxing Yu contributed equally to the work.

\section{Acknowledgments}

All authors would like to thank the participants of the study. They also thank Jiang Xiaolong for correcting the manuscript. This work was supported by the National Natural Science Foundation of China (Grant nos. 81873909 and 81873263).

\section{References}

[1] R. Solomon and S. Goldstein, "Real-time measurement of glomerular filtration rate," Current Opinion in Critical Care, vol. 23, no. 6, pp. 470-474, 2017.

[2] GBD Chronic Kidney Disease Collaboration, "Global, regional, and national burden of chronic kidney disease, 19902017: a systematic analysis for the Global Burden of Disease Study 2017," Lancet, vol. 395, no. 10225, pp. 709-733, 2020.

[3] K. J. Foreman, N. Marquez, A. Dolgert et al., "Forecasting life expectancy, years of life lost, and all-cause and cause-specific mortality for 250 causes of death: reference and alternative scenarios for 2016-40 for 195 countries and territories," The Lancet, vol. 392, no. 10159, pp. 2052-2090, 2018.

[4] B. Meijers, P. Evenepoel, and H.-J. Anders, "Intestinal microbiome and fitness in kidney disease," Nature Reviews Nephrology, vol. 15, no. 9, pp. 531-545, 2019.

[5] W. L. Lau, J. Savoj, M. B. Nakata, and N. D. Vaziri, "Altered microbiome in chronic kidney disease: systemic effects of gutderived uremic toxins," Clinical Science, vol. 132, no. 5, pp. 509-522, 2018.

[6] N. D. Vaziri, J. Yuan, S. Nazertehrani, Z. Ni, and S. Liu, "Chronic kidney disease causes disruption of gastric and small intestinal epithelial tight junction," American Journal of Nephrology, vol. 38, no. 2, pp. 99-103, 2013.

[7] M. Rossi, K. L. Campbell, D. W. Johnson et al., "Proteinbound uremic toxins, inflammation and oxidative stress: a cross-sectional study in stage 3-4 chronic kidney disease," Archives of Medical Research, vol. 45, no. 4, pp. 309-317, 2014.

[8] K. Shi, F. Wang, H. Jiang et al., "Gut bacterial translocation may aggravate microinflammation in hemodialysis patients," Digestive Diseases and Sciences, vol. 59, no. 9, pp. 2109-2117, 2014.
[9] R. Al-Sadi, M. Boivin, and T. Ma, "Mechanism of cytokine modulation of epithelial tight junction barrier," Frontiers in Bioscience, vol. 14, pp. 2765-2778, 2009.

[10] F. Wang, H. Jiang, K. Shi, Y. Ren, P. Zhang, and S. Cheng, "Gut bacterial translocation is associated with microinflammation in end-stage renal disease patients," Nephrology, vol. 17, no. 8, pp. 733-738, 2012.

[11] C. J. Lin, "Meta-analysis of the associations of p-cresyl sulfate (PCS) and indoxyl sulfate (IS) with cardiovascular events and all-cause mortality in patients with chronic renal failure," PLoS One, vol. 10, no. 7, Article ID e0132589, 2015.

[12] Y. H. Kim, "Indoxyl sulfate promotes apoptosis in cultured osteoblast cells," BMC Pharmacol Toxicol, vol. 14, p. 60, 2013.

[13] Y. Wu, D. Wang, X. Yang, C. Fu, L. Zou, and J. Zhang, "Traditional Chinese medicine Gegen Qinlian decoction ameliorates irinotecan chemotherapy-induced gut toxicity in mice," Biomedicine \& Pharmacotherapy, vol. 109, pp. 22522261, 2019.

[14] Y. Zang, J. Wan, Z. Zhang et al., "An updated role of astragaloside IV in heart failure," Biomed Pharmacother, vol. 126, Article ID 110012, 2020.

[15] L. Mei Ying, "The clinical effect of Fushen Granules in the treatment of intestinal dysfunction in peritoneal dialysis," China Medical Herald, vol. 13, no. 9, pp. 173-176, 2016.

[16] C. Jiang, W. Lin, L. Wang et al., "Fushen Granule, A Traditional Chinese Medicine, ameliorates intestinal mucosal dysfunction in peritoneal dialysis rat model by regulating p38MAPK signaling pathway," Journal of Ethnopharmacology, vol. 251, Article ID 112501, 2020.

[17] J. Ru, P. Li, J. Wang et al., "TCMSP: a database of systems pharmacology for drug discovery from herbal medicines," Journal of Cheminformatics, vol. 6, p. 13, 2014.

[18] UniProt Consortium, "UniProt: a hub for protein information,” Nucleic Acids Res, vol. 43, pp. D204-D212, 2015.

[19] G. R. Brown, V. Hem, K. S. Katz et al., "Gene: a gene centered information resource at NCBI," Nucleic Acids Research, vol. 43, no. D1, pp. D36-D42, 2015.

[20] J. Piñero, À. Bravo, N. Queralt-Rosinach et al., "DisGeNET: a comprehensive platform integrating information on human disease-associated genes and variants," Nucleic Acids Research, vol. 45, no. D1, pp. D833-d839, 2017.

[21] D. S. Wishart, "DrugBank: a comprehensive resource for in silico drug discovery and exploration," Nucleic Acids Research, vol. 34, no. 90001, pp. D668-D672, 2006.

[22] F. Zhu, B. Han, P. Kumar et al., "Update of TTD: therapeutic target database," Nucleic Acids Research, vol. 38, no. suppl_1, pp. D787-D791, 2010.

[23] N. Wang, F. Zhu, M. Shen et al., "Network pharmacologybased analysis on bioactive anti-diabetic compounds in Potentilla discolor bunge," Journal of Ethnopharmacology, vol. 241, Article ID 111905, 2019.

[24] P. Shannon, "Cytoscape: a software environment for integrated models of biomolecular interaction networks," Genome Research, vol. 13, no. 11, pp. 2498-2504, 2003.

[25] J. Zhang, X. Liu, J. Wu et al., "A bioinformatics investigation into the pharmacological mechanisms of the effect of the Yinchenhao decoction on hepatitis $\mathrm{C}$ based on network pharmacology," BMC Complementary Medicine and Therapies, vol. 20, no. 1, p. 50, 2020.

[26] R. Wang and J. Lin, "Analysis of the mechanism of zhichuanling oral liquid in treating bronchial asthma based on network pharmacology," Evidence-Based Complementary and Alternative Medicine, vol. 2020, Article ID 1875980, 10 pages, 2020. 
[27] Y. W. Sun, Y. Wang, Z.-F. Guo et al., "Systems pharmacological approach to investigate the mechanism of ohwia caudata for application to alzheimer's disease," Molecules, vol. 24 , no. $8,2019$.

[28] G. G. Loots, P. S. Chain, S. Mabery, A. Rasley, E. Garcia, and I. Ovcharenko, "Array2BIO: from microarray expression data to functional annotation of co-regulated genes," BMC Bioinformatics, vol. 7, p. 307, 2006.

[29] Y. Zhuang, H. Peng, V. Mastej, and W. Chen, "MicroRNA regulation of endothelial junction proteins and clinical consequence," Mediators Inflamm, vol. 2016, Article ID 5078627, 6 pages, 2016.

[30] G. W. Moeckel, L. Zhang, A. B. Fogo, C.-M. Hao, A. Pozzi, and M. D. Breyer, "COX2 activity promotes organic osmolyte accumulation and adaptation of renal medullary interstitial cells to hypertonic stress," Journal of Biological Chemistry, vol. 278, no. 21, pp. 19352-19357, 2003.

[31] Z. Jia, Y. Zhang, G. Ding et al., "Role of COX-2/mPGES-1/ prostaglandin E2 cascade in kidney injury," Mediators of Inflammation, vol. 2015, Article ID 147894, 8 pages, 2015.

[32] C.-M. Hao and M. D. Breyer, "Physiologic and pathophysiologic roles of lipid mediators in the kidney," Kidney International, vol. 71, no. 11, pp. 1105-1115, 2007.

[33] W. He, M. Zhang, M. Zhao et al., "Increased dietary sodium induces $\mathrm{COX} 2$ expression by activating $\mathrm{NF} \kappa \mathrm{B}$ in renal medullary interstitial cells," Pflügers Archiv-European Journal of Physiology, vol. 466, no. 2, pp. 357-367, 2014.

[34] L. Feng, J Su, R Chi, Q Zhu, S Lv, and W Liang, "Effect of amlodipine besylate combined with acupoint application of traditional Chinese medicine nursing on the treatment of renal failure and hypertension by the PI3K/AKT pathway," International Journal of Molecular Medicine, vol. 43, no. 4, pp. 1900-1910, 2019.

[35] D. Wu, N. Luo, L. Wang et al., "Hydrogen sulfide ameliorates chronic renal failure in rats by inhibiting apoptosis and inflammation through ROS/MAPK and NF- $\kappa$ B signaling pathways," Scientific Reports, vol. 7, no. 1, p. 455, 2017.

[36] G. Cheng, X. Tang, and J. Zhang, "Hepatocyte growth factor exerts beneficial effects on mice with type II diabetes-induced chronic renal failure via the NF- $\kappa \mathrm{B}$ pathway," Molecular Medicine Reports, vol. 18, no. 3, pp. 3389-3396, 2018.

[37] Y. Wang, L. Zhang, H. Jin et al., "Based on HIF-1 $\alpha /$ Wnt/ $\beta$-Catenin pathway to explore the effect of qingshen granules on chronic renal failure patients: a randomized controlled trial," Evidence-Based Complementary and Alternative Medicine, vol. 2019, Article ID 7656105, 7 pages, 2019.

[38] L. Cuarental, D. Sucunza-Sáenz, L. Valiño-Rivas et al., "MAP3K kinases and kidney injury," Nefrología, vol. 39, no. 6, pp. 568-580, 2019.

[39] M. Zhang, Y. Chen, M. J. Yang et al., "Celastrol attenuates renal injury in diabetic rats via MAPK/NF- $\kappa$ B pathway," Phytotherapy Research, vol. 33, no. 4, pp. 1191-1198, 2019.

[40] Y. Feng, J. Ren, Y. Gui et al., "Wnt $/ \beta$-catenin-promoted macrophage alternative activation contributes to kidney fibrosis," Journal of the American Society of Nephrology, vol. 29, no. 1, pp. 182-193, 2018.

[41] T. Kawakami, S. Ren, and J. S. Duffield, "Wnt signalling in kidney diseases: dual roles in renal injury and repair," The Journal of Pathology, vol. 229, no. 2, pp. 221-231, 2013.

[42] E. D. Arisan, R. O. Akar, O. Rencuzogullari et al., "The molecular targets of diclofenac differs from ibuprofen to induce apoptosis and epithelial mesenchymal transition due to alternation on oxidative stress management p53 independently in PC3 prostate cancer cells," Prostate International, vol. 7, no. 4, pp. 156-165, 2019.

[43] A. Mantovani, "The inflammation-cancer connection," The FEBS Journal, vol. 285, no. 4, pp. 638-640, 2018.

[44] J. Chen, "Curative effect observation of TCM intervention of intestinal barrier dysfunction in CAPD," Tianjin Journal of Traditional Chinese Medicine, vol. 31, no. 08, pp. 462-465, 2014.

[45] E. Hoibian, N. Florens, L. Koppe et al., "Distal colon motor dysfunction in mice with chronic kidney disease: putative role of uremic toxins," Toxins, vol. 10, no. 5, 2018.

[46] P.-S. Hsieh, K.-C. Lu, C.-F. Chiang, and C.-H. Chen, "Suppressive effect of COX2 inhibitor on the progression of adipose inflammation in high-fat-induced obese rats," European Journal of Clinical Investigation, vol. 40, no. 2, pp. 164-171, 2010.

[47] S. Satta, A. M. Mahmoud, F. L. Wilkinson et al., "The role of $\mathrm{Nrf} 2$ in cardiovascular function and disease," Oxidative Medicine and Cellular Longevity, vol. 2017, Article ID 9237263, 18 pages, 2017.

[48] A. Loboda, M. Damulewicz, E. Pyza, A. Jozkowicz, and J. Dulak, "Role of Nrf2/HO-1 system in development, oxidative stress response and diseases: an evolutionarily conserved mechanism," Cellular and Molecular Life Sciences, vol. 73, no. 17, pp. 3221-3247, 2016.

[49] S. H. Aladaileh, O. E. Hussein, M. H. Abukhalil et al., "Formononetin upregulates $\mathrm{Nrf2} / \mathrm{HO}-1$ signaling and prevents oxidative stress, inflammation, and kidney injury in Methotrexate-Induced rats," Antioxidants, vol. 8, no. 10, 2019.

[50] Y.-M. Jin, X.-M. Tao, Y.-N. Shi, Y. Lu, and J.-Y. Mei, "Salvianolic acid $\mathrm{B}$ exerts a protective effect in acute liver injury by regulating the Nrf2/HO-1 signaling pathway," Canadian Journal of Physiology and Pharmacology, vol. 98, no. 3, pp. 162-168, 2020.

[51] T. Cakir, B. Goktas, M. F. Mutlu et al., "Advanced oxidation protein products and malondialdehyde-the new biological markers of oxidative stress-are elevated in postmenopausal women," Ginekologia Polska, vol. 87, no. 5, pp. 321-325, 2016. 